\title{
Masers in Active Galactic Nuclei
}

\section{J. Greenhill}

Harvard-Smithsonian Center for Astrophysics, 60 Garden St, Cambridge, MA 02138, USA

\begin{abstract}
Studies of $\mathrm{OH}$ and $\mathrm{H}_{2} \mathrm{O}$ masers contribute to our understanding of the dynamics and physical conditions in the innermost few parsecs of AGN. Angular and velocity resolutions that may be routinely achieved with radio interferometry are substantially better than those possible with traditional observational techniques in the optical and ultraviolet.
\end{abstract}

\section{Introduction}

Four species are known to exhibit maser action in galactic nuclei: $\mathrm{H}_{2} \mathrm{O}, \mathrm{OH}$, $\mathrm{H}_{2} \mathrm{CO}$, and $\mathrm{CH}$ (Henkel et al. 1991 and references therein). Typically, extragalactic $\mathrm{H}_{2} \mathrm{O}$ masers at $1 \mathrm{~cm}$ wavelength are up to two orders of magnitude stronger than $\mathrm{OH}$ masers at $18 \mathrm{~cm}$, which are up to two orders of magnitude stronger than the $\mathrm{H}_{2} \mathrm{CO}$ and $\mathrm{CH}$ masers at 6 and $9 \mathrm{~cm}$, respectively. $\mathrm{OH}$ and $\mathrm{H}_{2} \mathrm{O}$ masers have been studied most thoroughly with high angular resolution. Henkel et al. (1991) and Baan et al. (1993) review $\mathrm{H}_{2} \mathrm{CO}$ and $\mathrm{CH}$ masers (see also Baan \& Haschick 1995).

Masers are point-like tracers of the dynamics and physical conditions within the dense molecular gas of galactic nuclei because (1) their spectra consist of many individual Doppler components, $(2)$ these components subtend small solid angles on the sky (e.g., $<10^{-6} \operatorname{arcsec}^{2}$ for $\mathrm{H}_{2} \mathrm{O}$ ), and (3) the microwave emission is not subject to extinction by dust along the line of sight. Masers are suited to study with interferometry because of their small angular size and high surface brightness, which makes routinely possible angular resolutions of $\sim 0.5$ milliarcseconds (mas) at $1 \mathrm{~cm}$ wavelength $(0.1 \mathrm{pc}$ at $40 \mathrm{Mpc})$ and velocity resolutions of $\sim 0.5 \mathrm{~km} \mathrm{~s}^{-1}$. On the other hand, masers require (1) relatively quiescent conditions for the survival of molecular gas, (2) a coherent velocity field, to support amplification, and (3) an observer who is situated in the path of the maser emission, which is often anisotropic. Maser emission is visible to us for only a fraction of those galaxies that have masers. (Anisotropic emission arises from an elongated maser cloud and from background amplification by a non-spherically symmetric collection of maser clouds.)

\section{Extragalactic OH Masers}

Extragalactic $\mathrm{OH}$ masers occur in infrared-luminous galaxies $\left(L_{\mathrm{IR}} \gtrsim 10^{11.5} \mathrm{ergs}\right.$ $\mathrm{s}^{-1}$ ), which Sanders et al. (1988) suggest may contain dust-enshrouded active 
galactic nuclei (AGN). There is circumstantial evidence that $\mathrm{OH}$ masers are associated with edge-on nuclear structures. Almost $40 \%$ of extragalactic $\mathrm{OH}$ masers are found in starburst nuclei, and almost $60 \%$ are found in Seyfert 2 nuclei and LINERs, which is disproportionately large compared to the fraction of AGN in the IRAS galaxy sample (Baan 1993). A few weak OH masers also occur in some lower-luminosity galaxies, where strong $\mathrm{OH}$ absorption occurs as well, though the sample of galaxy types is rather heterogeneous.

In early models, $\mathrm{OH}$ maser emission came from clouds in circumnuclear structures on scales of at least $100 \mathrm{pc}$, where they amplified nuclear radio continuum emission (e.g., Baan \& Haschick 1994). Recent observations argue for smaller size scales. The masers in Arp 220 (Diamond et al. 1989) and III Zw 35 (A. Trotter, personal communication) have been resolved into widely separated complexes each less than a few tens of pc in size. Many IR luminous galaxies are merger systems and these complexes probably mark separate nuclei. At higher resolution, most of the maser emission in Arp 220 actually arises on scales $<10 \mathrm{pc}$, including a sub-parsec scale component coincident with a continuum component $<10 \mathrm{pc}$ in size (Lonsdale et al. 1994). Based on these recent observations, a new model is emerging in which the spectral components of $\mathrm{OH}$ masers correspond to compact dense clumps of gas $\left(r<1 \mathrm{pc} ; n_{\mathrm{H}_{2}}=10^{5}-10^{7} \mathrm{~cm}^{-3}\right)$ within the innermost roughly $10 \mathrm{pc}$ of host AGN, though continued study at high angular resolution is clearly needed.

\section{Extragalactic $\mathrm{H}_{2} \mathrm{O}$ Masers}

Water maser emission is known to exist in 17 AGN: Mrk 1, NGC 1052, NGC 1068, NGC 1386, Mrk 1210, NGC 2639, NGC 3079, IC 2560, NGC 4258, NGC 4945, NGC 5347, the Circinus galaxy, NGC 5506, ESO 103-G35, IRAS 22265-1826, IC 1481 (Braatz et al. 1996), and M 51 (Ho et al. 1987). Of these, 15 are late-type galaxies containing Seyfert 2 nuclei or LINERs. Two are early-type galaxies, both of which contain LINERs and have distinctive broad-line shapes devoid of the narrow features found in the other maser sources (Fig. 1). Braatz et al. (1996) find that 5-10\% of AGN closer than $c z=7000 \mathrm{~km} \mathrm{~s}^{-1}$ contain $\mathrm{H}_{2} \mathrm{O}$ emission, which may be a consequence of anisotropic emission. I discuss in detail three of the maser sources that have been observed with Very Long Baseline Interferometry (VLBI).

\subsection{NGC 4258}

NGC 4258 is the best example of how masers may be used to accurately determine the geometry and dynamics within $1 \mathrm{pc}$ an AGN central engine. Nakai et al. (1993) discovered two complexes of emission, hereafter 'high-velocity emission', symmetrically offset by about $\pm 900 \mathrm{~km} \mathrm{~s}^{-1}$ from the emission near the systemic velocity of the galaxy, hereafter 'systemic emission' (Fig. 1). VLBI observations show that the masers trace a warped disk of 0.13 and $0.26 \mathrm{pc}$ inner and outer radii, respectively (Miyoshi et al. 1995). The high-velocity emission is distributed in radius and follows a Keplerian rotation law. The systemic emission lies close to the inner radius (Fig. 2). The line-of-sight accelerations of the high-velocity masers constrain them to lie very close to the diameter perpendicular to the line of sight. The acceleration of the systemic masers is consistent 


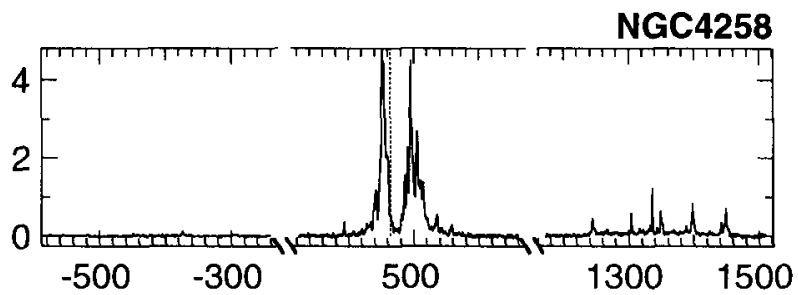

NGC 4945

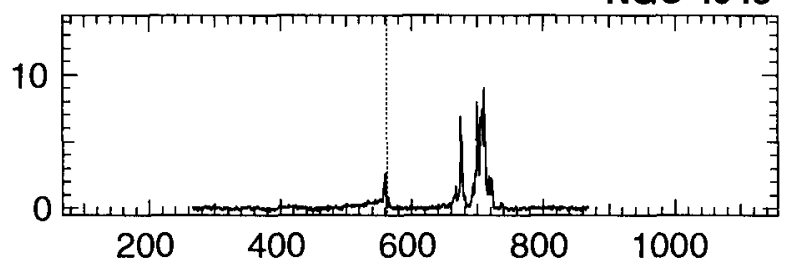

Circinus

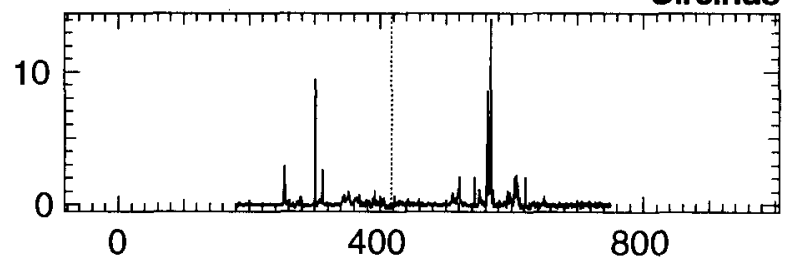

NGC 1068
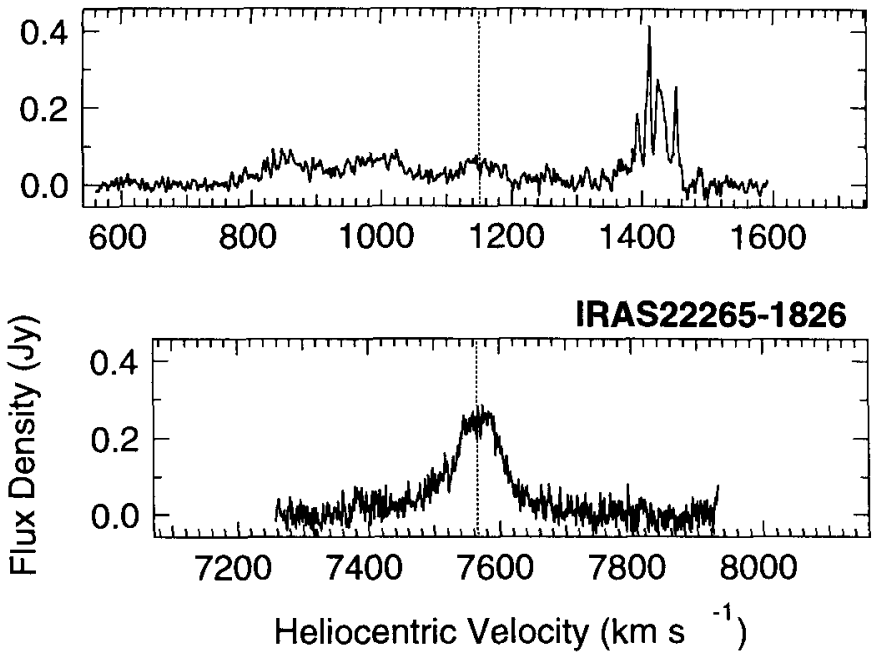

Figure 1. Spectra of several of the strongest AGN $\mathrm{H}_{2} \mathrm{O}$ masers, showing different morphologies, i.e., arrangements of emission complexes at the systemic velocities and at highly redshifted and blueshifted velocities. IRAS 22265-1826 is the only early-type galaxy shown (from Koekemoer et al. 1995). The velocity scales of the spectra are identical. The optical definition of radial velocity is assumed. 

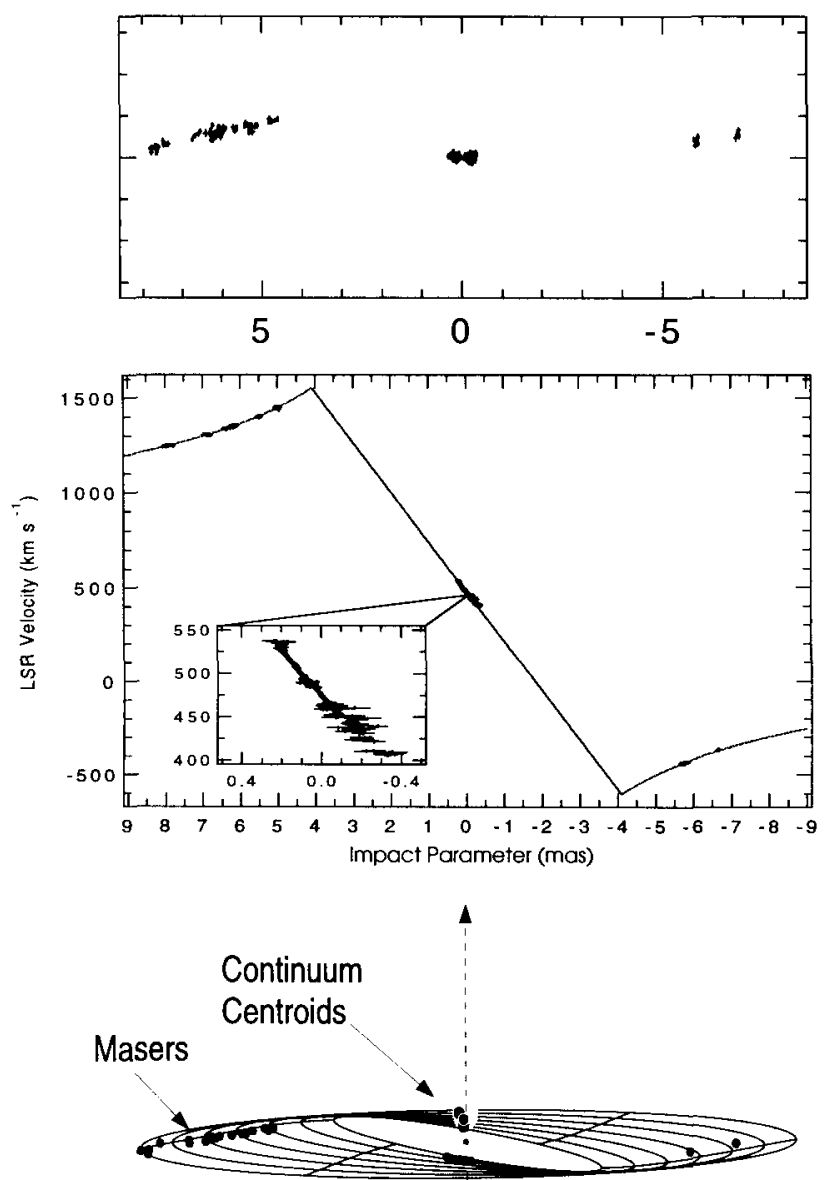

Figure 2. VLBI data and model for NGC 4258. Top: Distribution of maser emission on the sky. Errors are shown. At a distance of $6.4 \mathrm{Mpc}$, 5 mas corresponds to 0.15 pc. Middle: Position-velocity diagram showing Keplerian tails traced by the high-velocity emission (from Miyoshi et al. 1995). Bottom: Maser distribution sampling a thin, warped disk tipped down $8^{\circ}$ on the near side. The axis of the radio jet in the nucleus (observed on parsec scales) is roughly north-south. Additional spots mark the disk dynamical center (Herrnstein et al. 1996a), and the centroids of $1 \mathrm{~cm}$ continuum emission, observed at three epochs over two years (Herrnstein et al. 1996b). The position uncertainties for the dynamical center and centroids are comparable to the sizes of the respective symbols. The sizes of the continuum sources are less than around 1 mas. 
with their orbital velocity and radius, for a distance of about $6.4 \mathrm{Mpc}$ (Greenhill et al. 1995). The disk is very thin (i.e., thickness-to-radius ratio $<0.3 \%$ ) and may be in hydrostatic pressure equilibrium (Moran et al. 1995). The Eddington luminosity of the $3.5 \times 10^{7} M_{\odot}$ central mass is $\sim 10^{4}$ times greater than the observed X-ray luminosity of the AGN (Makishima et al. 1994). The thinness of the disk may be a consequence of the low central-engine luminosity.

High-resolution imaging of radio continuum emission in AGN usually is limited by assumptions about the location of the central engine. Herrnstein et al. (1996b) report the detection at $1 \mathrm{~cm}$ wavelength of the base of the well-known northern jet in NGC 4258 (Cecil et al. 1995, and references therein) and are able to register it with respect to the disk center, to a few $\times 10^{-3} \mathrm{pc}$ (Fig. 2). (This is possible because both position measurements are referenced to the maser emission.) The masers lie at $\sim 5 \times 10^{4}$ gravitational radii, and the jet emission lies at only $\sim 5 \times 10^{3}$ radii. The strength of the jet emission varies significantly on time scales of 1 week and its location changes over 2 years. This emission is at least ten times stronger than any emission that might be associated specificially with the central engine.

\subsection{NGC 1068}

Water-maser emission in NGC 1068 extends about $\pm 300 \mathrm{~km} \mathrm{~s}^{-1}$ from the systemic velocity (Fig. 1). Based on the first VLBI images of the maser (Fig. 3), Greenhill et al. (1996) suggest that the maser traces part of the limb of a molecular torus that surrounds the central engine. The torus is axially thick, with comparable height and radius. The maser traces sub-Keplerian differential rotation $\left(v \propto r^{-0.3}\right)$ and argues for a central mass of $\sim 10^{7} M_{\odot}$, within a cylindrical radius of about $0.65 \mathrm{pc}$, at a distance of $15 \mathrm{Mpc}$. The estimated luminosity of the engine (Pier et al. 1994) is about 0.5 of the Eddington limit.

A thin disk model is less likely for NGC 1068 than for NGC 4258 because (1) the orientation of the arc of red-shifted maser emission is not perpendicular to the axis of the known radio jet (Ulvestad et al. 1987, Muxlow et al. 1996) and (2) the average centroid of blue-shifted maser emission lies due east of the centroid for red-shifted emission (Gallimore et al. 1996). The blueshifted emission has only been imaged with at $\sim 0^{\prime \prime} .1$ resolution with the VLA. Hence, the redshifted emission can be reflected about the systemic velocity and the jet axis to yield a ' $V$ '-shaped structure on the sky, which may be interpreted as the limb of a thick torus (Fig. 3). The limb provides optimal velocity coherence in the molecular gas because the orbital velocities are parallel to the line of sight, for circular orbits. The limb is also exposed to possible sources of excitation, such as Xray illumination by the central engine (Nuefield et al. 1994) or shock interaction with the jet (Elitzur et al. 1989). The inner edge of the torus near the equatorial plane also provides optimal coherence, where orbital velocities are transverse to the line of sight.

There is no detectable compact radio continuum emission near the proposed center of the torus $\left(T_{B}<5 \times 10^{6} \mathrm{~K}\right.$ on size scales of $\left.\sim 0.1 \mathrm{pc}\right)$, so that the observed flat-spectrum core near the base of the jet (e.g., Gallimore, Baum, \& O'Dea 1996) cannot be direct self-absorbed synchrotron radiation. 

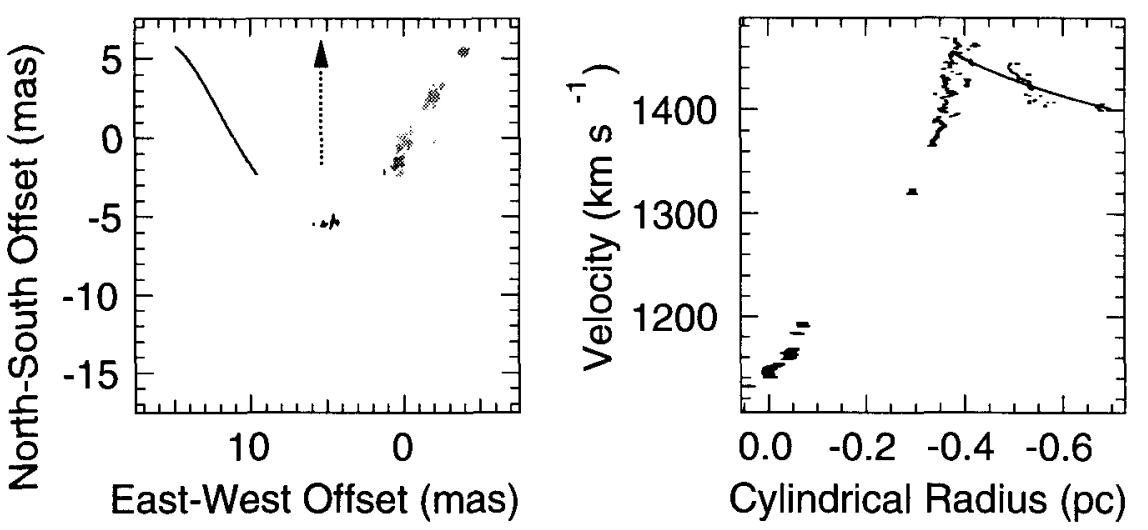

Figure 3. Left: Distribution of systemic and redshifted maser emission in NGC 1068 (from Greenhill et al. 1996a), where the grayscale reflects radial velocity. Systemic emission is darkest. The solid line shows the anticipated location of the blueshifted emission when it is observed with VLBI. The dashed line indicates the approximate axis of the radio jet on parsec scales. At a distance of $15 \mathrm{Mpc}, 0.7 \mathrm{pc}$ subtends 10 mas. Right: Position-velocity diagram, showing a sub-Keplerian tail and possibly turbulent velocities of up to $100 \mathrm{~km} \mathrm{~s}^{-1}$. The cylindrical radius is measured with respect to the maser feature at the systemic velocity along a position angle of $-90^{\circ}$.

\subsection{NGC 4945}

The nucleus of NGC 4945 is both a Seyfert 2 and starburst object (Iwasawa et al. 1993; Moorwood \& Oliva 1994, and references therein). The $\mathrm{H}_{2} \mathrm{O}$ maser source is important because it may be used to probe the structure and dynamics of this heavily obscured object on sub-parsec scales. Maser emission is present near the systemic velocity and also redshifted by $100-200 \mathrm{~km} \mathrm{~s}^{-1}$ (Fig. 1). No emission has been observed with a symmetric blueshift. This source is a challenge to study because the established very-long-baseline interferometers operating at $1 \mathrm{~cm}$ wavelength lie in the northern hemisphere and the galaxy's declination is $-49^{\circ}$.

Recent sub-arcsecond astrometry establishes that the maser lies within the inner $10 \mathrm{pc}$ of the galactic nucleus and probably marks the center (Greenhill et al. 1996). The maser emission extends over $\sim 0.4 \mathrm{pc}$ at a position angle of $\sim 45^{\circ}$, which is perpendicular to the ionization cone observed along the minor axis of the galaxy (Nakai 1989). Moreover, the systemic emission exhibits a positionvelocity gradient of $\sim 1.5 \mathrm{~km} \mathrm{~s}^{-1} \mathrm{mas}^{-1}$, which is an indicator of orbital motion around a central mass. The position-velocity gradient, linear structure, and proximity of the maser source to the galactic center suggest that it might trace part of an edge-on disk, with inner radius $\sim 0.2 \mathrm{pc}$ and central binding mass $\sim 10^{6} M_{\odot}$, assuming circular orbits. The orbital velocity at $0.2 \mathrm{pc}$ and the position-velocity gradient suggests the systemic masers would lie at an outer radius of $\sim 0.9 \mathrm{pc}$ (Greenhill et al. 1996). The central engine radiates about 

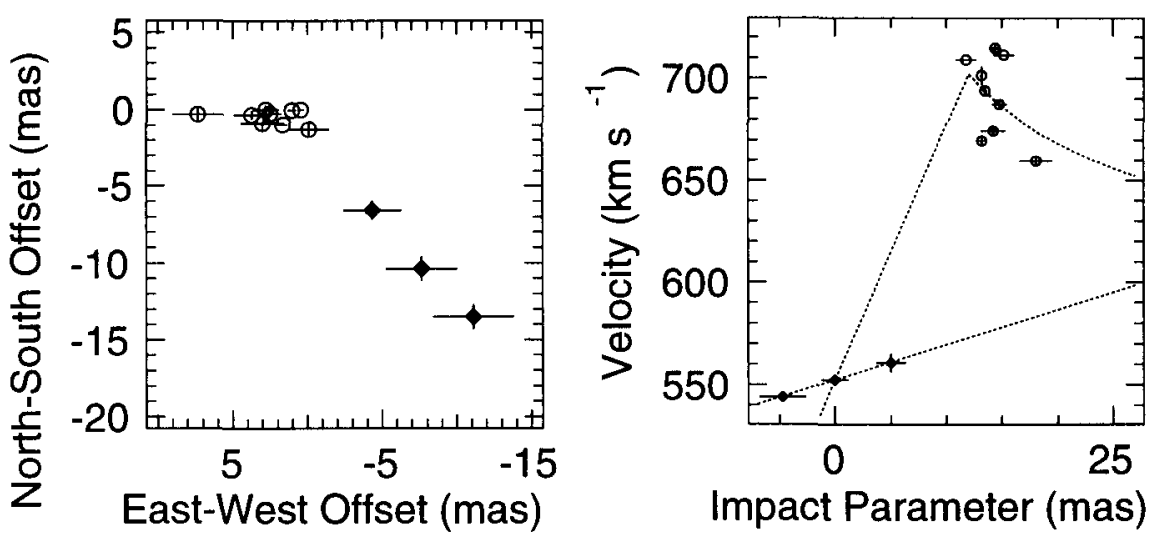

Figure 4. Left: Distribution of maser emission in NGC 4945 (from Greenhill et al. 1996b). Filled and open circles indicate systemic and redshifted emission, respectively. The position angle of the galactic disk is about $45^{\circ}$. Right: Position-velocity diagram for the maser emission. The impact parameter is measured from the maser feature closest to the systemic velocity. The dashed line is the boundary within which maser emission should lie if they are confined to an annular disk. Features on the upper and lower straight lines would lie along the inner and outer radii, respectively. Masers on the Keplerian curve would lie on the radius perpendicular to the line of sight.

$10 \%$ of its Eddington luminosity if its bolometric luminosity is 10 times its $\mathrm{X}$ ray luminosity (Iwasawa et al. 1993). Admittedly, this disk interpretation is not unique and radically different interpretations might be viable.

\section{References}

Baan, W.A. 1993, in Sub-Arcsecond Radio Astronomy, eds. R. J. Davis \& R.S. Booth, (Cambridge Univ. Press: Cambridge), 324.

Baan, W. A., \& Haschick, A. D. 1984, ApJ, 279, 541.

Baan, W.A., Haschick, A. D., \& Uglesich, R. 1993, ApJ, 415, 140.

Baan, W. A., \& Haschick, A. D. 1995, ApJ, 454, 745.

Braatz, J. A., Wilson, A.S., \& Henkel, C. 1996, ApJS, 106, in press.

Cecil, G., Wilson, A.S., \& de Pree, C. 1995, ApJ, 440, 181.

Diamond, P. J., Norris, R. P., Baan, W. A., \& Booth, R. S. 1989, ApJ, 340, L49.

Elitzur, M., Hollenbach, D. J., \& McKee, C.F. 1989, ApJ, 346, 983.

Gallimore, J.F., Baum, S. A., \& O'Dea, C. P. 1996a, ApJ, 464, 198.

Gallimore, J.F., Baum, S.A., O'Dea, C.P., Brinks, E., \& Pedlar, A. 1996b, ApJ, 462, 740 . 
Greenhill, L. J., Henkel, C., Becker, R., Wilson, T.L., \& Wouterloot, J. G.A. 1995, A\&A, 304, 21.

Greenhill, L. J., Gwinn, C.R., Antonucci, R., \& Barvainis, R. E. 1996, ApJ, in press.

Greenhill, L. J., Moran, J. M., \& Herrnstein, J. R. 1996, ApJ, submitted.

Henkel, C., Baan, W.A., \& Mauersberger, R. 1991, A\&A Rev. 3, 47.

Herrnstein, J. R., Greenhill, L. J., \& Moran, J. M. 1996a, ApJ, in press.

Herrnstein, J.R., Moran, J.M., Greenhill, L.J., Diamond, P.J., Miyoshi, M., Nakai, N., \& Inoue, M. 1996b, ApJ, submitted.

Ho, P. T.P., Martin, R. N., Henkel, C., \& Turner, J. L. 1987, Ap.J., 320, 663.

Iwasawa, K., et al. 1993, ApJ, 409, 155.

Koekemoer, A. M., Henkel, C., Greenhill, L.J., Dey, A., van Breugel, W., \& Antonucci, R. 1995, Nature, 378, 697.

Lonsdale, C., Diamond, P., Smith, H., \& Lonsdale, C. 1994, Nature, 370, 117.

Makishima, K., et al. 1994, PASJ, 46, L77.

Miyoshi, M., Moran, J.M., Herrnstein, J. R., Greenhill, L.J., Nakai, N., Diamond, P. J., \& Inoue, M. 1995, Nature, 373, 127.

Moorwood, A. F. M., \& Oliva, E. 1994, ApJ, 429, 602.

Moran, J. M., Greenhill, L. J., Herrnstein, J. R., Diamond, P.J., Nakai, N., Inoue, M., \& Miyoshi, M., Proc. Nat. Acad. Sci. USA, 92, 11427.

Muxlow, T.W.B., Pedlar, A., Holloway, A., Gallimore, J.F., \& Antonucci, R. R. J. 1996, MNRAS, $278,854$.

Nakai, N. 1989, PASJ, 41, 1107.

Nakai, N., Inoue, M., \& Miyoshi, M. 1993, Nature, 361, 45.

Neufeld, D. A., Maloney, P.R., \& Conger, S. 1994, ApJ, 436, L127

Pier, E. A., Antonucci, R. R.J., Hurt, T., Kriss, G., \& Krolik, J. 1994, ApJ, 428, 124.

Sanders, D. B., Soifer, B.T., Elias, J.H., Madore, B.F., Matthews, K., Neugebauer, G., \& Scoville, N.Z. 1988, ApJ, 325, 74.

Ulvestad, J. S., Neff, S. G., \& Wilson, A.S. 1987, AJ, 93, 22. 